\title{
Auto-antibodies shape brain development in myriad ways
}

\author{
BY ANGIE VOYLES ASKHAM
}

8 NOVEMBER 2021

Prenatal exposure to maternal antibodies may contribute to autism in various ways depending on the antibodies involved, new unpublished studies suggest.

Two teams of researchers presented the findings virtually at the 2021 Society for Neuroscience annual meeting.

A pregnant woman's antibodies can pass to her developing fetus through the placenta. Though most antibodies are beneficial and help protect the fetus from pathogens, certain forms - called 'auto-antibodies' - can also attack its brain cells and alter its development.

Women who have autistic children are more likely to have these auto-antibodies than are women who have non-autistic children, previous studies show. And mice, rats and monkeys exposed to those autism-linked auto-antibodies in the womb show some traits reminiscent of autism.

The two new lines of work hint at the underlying mechanisms.

\section{Active cells:}

In one study, researchers prompted female mice to produce auto-antibodies by repeatedly injecting them with a portion of a protein called CASPR2, which is encoded by the autism-linked gene CNTNAP2. These animals' male offspring, but not their female offspring, showed autism-like behaviors such as repetitive behaviors and decreased sociability, the researchers found.

The male offspring also had atypical neurons in their hippocampus, a brain region involved in learning and memory. Compared with those of female offspring and controls, the exposed males' cells had sparser dendrites, the arms of a neuron that receive signals from other cells, as well as fewer dendritic spines, the nodes at which synapses between cells form. 


\section{Spectrum | Autism Research News}

https://www.spectrumnews.org

Some dendritic pruning, mediated by brain cells called microglia, is necessary for typical brain development. But it seems to have gone into overdrive for the exposed male mice, the team found: The animals had an unusually high density of microglia in their hippocampus. And their microglia were thick, with shorter processes - a hallmark of activated cells - rather than being thin with many spindly arms, as in the control animals and the exposed females.

Microglia are activated during brain development and after brain injury, but it's unclear what prompts the activation in the model animals' cells, says lead investigator Lior Brimberg, assistant professor of neuroimmunology at the Feinstein Institutes for Medical Research in Manhasset, New York.

Treating the exposed male mice with the drug captopril, which is thought to suppress microglial activation, eliminated the differences in their microglia and normalized the appearance of their hippocampal neurons.

"There was a noticeable effect between the [levels of microglial] activation and also the dendritic branching" in mice that received the drug versus saline, says Ben Spielman, a graduate student in Brimberg's lab and the Donald and Barbara Zucker School of Medicine at Hofstra/Northwell in Hempstead, New York, who presented the work.

Shape shift: Microglia from male mice exposed to auto-antibodies in the womb are thick with short processes (top right), unlike those of their female counterparts (bottom right) and control mice (left).

\section{Reconciling differences:}

In the other new study, a different team injected female rats with a cocktail of peptides that are targets of the auto-antibodies found in some women who have autistic children. The researchers then bred these rats after they generated their own auto-antibodies against the peptides.

Like the mice in Brimberg's study, the offspring of these rats exhibited autism-like traits, including decreased communication and sociability and increased repetitive behaviors. The rats also had atypical brain development: Exposed males had changes to their sensory cortex, and exposed animals of both sexes had atypical structure in their cerebellum and midbrain.

The model animals also differed from controls in some of the metabolites present in their brain, the researchers found using magnetic resonance spectroscopy. Compared with controls, rats exposed to the auto-antibodies in utero had higher levels of taurine and lower levels of choline, which may contribute to atypical development by upsetting a balance between neuronal excitation and inhibition or causing changes in myelination, says Matthew Bruce, a graduate student in Judy 


\section{Spectrum | Autism Research News}

https://www.spectrumnews.org

Van de Water's lab at the University of California, Davis, who presented the work.

Bruce and his colleagues next plan to study how these antibodies bind to neurons in culture and whether they are being taken up into the cells. "That's the biggest future direction - understanding what is happening here at the cellular level," Bruce says.

As for the differences in the two auto-antibody models of autism, such as the sex differences seen in animals exposed to CASPR2 auto-antibodies but not those given the auto-antibody cocktail, Bruce says the field can reconcile them.

Brimberg agrees. The different models may be associated with different autism subtypes, she suggests. "I'm hoping someday in the future we'll have enough data, enough knowledge" to be able to link them together, she says.

Read more reports from the 2021 Society for Neuroscience virtual annual meeting.

Cite this article: https://doi.org/10.53053/FLXF9488 